\title{
GnRH analogues: applications in assisted reproductive techniques
}

\author{
Catherine Hayden \\ Reproductive Medicine Unit, The Leeds Teaching Hospitals NHS Trust, Clarendon Wing, Belmont Grove, Leeds LS2 9NS, UK \\ (Correspondence should be addressed to C Hayden; Email: catherinejhayden@hotmail.com)
}

\begin{abstract}
The ability to prevent an endogenous LH surge revolutionised the efficacy of assisted reproductive techniques (ART) such that GnRH agonists were rapidly adopted in the 1980s. Prior to this, premature luteinisation occurred in up to $25 \%$ of superovulated cycles leading to cycle cancellation and severely compromised outcomes. Analogues have been applied in a variety of drug protocols (long, short flare) but there has been little research to moderate the degree of pituitary suppression. There has also been ongoing and unresolved debate about the role of LH in supporting follicular development.

By 2001, the first GnRH antagonists were registered for use in ART. Their ability to cause immediate suppression of gonadotrophin (particularly LH) secretion means that they can be given after exogenous stimulation has begun and thereby dramatically shorten the total duration of a treatment cycle. After initial enthusiasm and then scepticism that pregnancy rates may not be as high as the established agonist regimens, these preparations are now being increasingly adopted with at least comparable outcomes in large trials. They are certainly favoured by patients for their reduced side-effect profile and particularly for the shortening of the total cycle length. This shift in practice is occurring alongside gathering momentum in favour of milder stimulation protocols and a new perception of what constitutes successful treatment. The focus is moving away from surrogate outcomes such as oocyte numbers and conception rates towards long-term outcomes for women and their offspring, namely the achievement of a live singleton birth per treatment started.
\end{abstract}

European Journal of Endocrinology 159 S17-S25

\section{Historical perspective}

The journey to our current understanding of GnRH began in the early 20th century when it was discovered that lesions of the anterior pituitary gave rise to genital atrophy, thereby identifying the pituitary-gonadal axis. By 1928, Ascheim and Zondek (1) demonstrated the stimulation of gonadal function with urine derived from pregnant women. Within 3 years, Fevold provided the first convincing evidence of two gonadotrophins (2), leading to the subsequent purification of FSH and LH. Over the next 30 years, ovarian stimulation with exogenous preparations including i.v. pregnant mare's serum (3) and human pituitary gland extracts (4) were attempted with limited success due to antibody formation and safety concerns. By the 1960s, technology was sufficiently advanced to extract FSH and LH from the urine of postmenopausal women although there was still significant contamination with impurities (proteins with allergic potential) causing batch to batch variability and poorly

This paper was presented at the 5th Ferring International Paediatric Endocrinology Symposium, Baveno, Italy (2008). Ferring Pharmaceuticals has supported the publication of these proceedings. predictive responses (5). Nevertheless, these preparations became routinely used to stimulate ovarian function.

The GnRH decapeptide was isolated and its structure elucidated in 1971 (Fig. 1). Amino acid substitutions have revealed the significance of specific regions for stability, receptor binding and activation of the pituitary gonadotrophs (6). Modifications mainly at positions 6 and 10 gave rise to analogues with increased potency, extending the half life from minutes to hours and raising the binding capacity more than 100 -fold.

The phenomenon of an initial flare in response to GnRH agonist (GnRH1a) followed by downregulation of the pituitary GnRH receptors by clustering and internalisation was clarified by 1978 (7). In 1980, pulsatile low-dose GnRH1a was used in a physiological manner to produce the first reported pregnancy in a woman with hypogonadotrophic hypogonadism (8). Through the early 1980s, GnRH1a was applied to prevent the LH surge during ovarian stimulation (9). By the end of the decade, the so-called long downregulation protocol with GnRH1a was established IVF practice, significantly reducing the rate of cycle cancellation, improving outcomes and enabling some flexibility to schedule oocyte retrieval (10). (Fig. 2a). There followed a vast expansion of IVF practice and provision. 


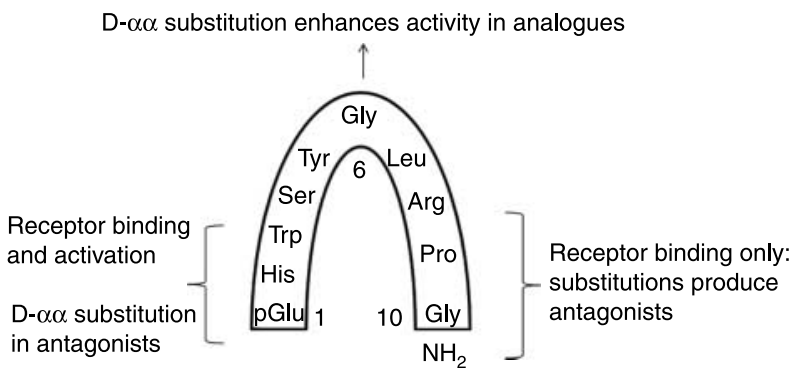

Figure $1 \mathrm{GnRH}$ schematic. Native $\mathrm{GnRH}$ is folded at Gly position 6 when it is bound to the pituitary receptor. Substitutions at position 6 stabilise the folded conformation and decrease metabolic clearance; all agonists and antagonists have this feature, e.g. Leuprorelin (Wyeth, Maidenhead, UK) has D Leu, Zoladex (AstraZeneca Luton, UK) has D Ser, Ganirelix (Oganon Cambridge, UK) Dh Arg agonists often also have substitutions at position 10 . Antagonists are usually modified at positions 1,2,3,6 and 10. The half-life of native $\mathrm{GnRH}$ is minutes. The agonist half-lives are hours. Receptor binding triggers receptor dimerisation and the cascade of intracellular events leading to synthesis/secretion of $\mathrm{FSH} / \mathrm{LH}$. The dimer is internalised after the signal has been passed on, but is unable to return to the membrane as long as the membrane receptors remain occupied. The cell is then unresponsive to further $\mathrm{GnRH}$ signal. Antagonists competitively bind to the receptor and stop dimer formation and signal transduction in a dose-dependent manner. They do not deplete FSH/LH supply and function may therefore be rapidly resumed. Reproduced and modified with permission from Prof. R.P. Millar MRC Human Reproductive Sciences Unit, Centre for Reproductive Biology, Edinburgh, UK.

It took a considerable period of time after the elucidation of GnRH to generate clinically useful antagonists (GnRH ant). The third generation compounds (Ganirelix (Organon, Cambridge, UK) and Cetrorelix (Merck-Serono, Feltham, UK)) reached the market in 2001. Their ability to induce immediate suppression and subsequent rapid recovery of pituitary function is particularly appropriate to their short term use in IVF.

Standard IVF treatment entails controlled ovarian stimulation of multiple follicle development that allows for inefficiencies further down the treatment line such as oocyte maturation in vitro, fertilisation in vitro, embryo culture, embryo selection and implantation. The subsequent transfer of multiple embryos and

(a)

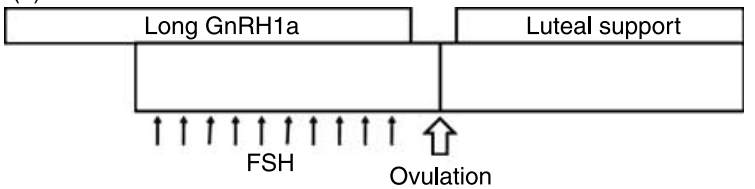

(b)

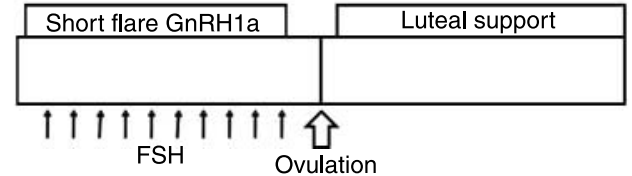

Figure $2 \mathrm{GnRH}$ agonist protocols. a) Long agonist downregulation starting in mid-luteal phase of previous cycle. b) Short flare agonist downregulation starting on day 2 of menses, combining endogenous FSH peak with agonist flare response to maximise recruitment. cryopreservation of good quality spare embryos maximises the efficiency of the stimulated cycle. Whilst the goal of treatment has always been a healthy live birth, the inefficiencies of the process have led to surrogate markers being used as measures of success, such as the number of follicles recruited, the number of oocytes retrieved/fertilised, the number of embryos created, the number of positive pregnancy tests/clinical pregnancies (foetal heart positive) identified.

The potential for IVF success is influenced by an infinite number of factors including clinical/biological aspects, pharmacological manipulations, technological aspects and laboratory practices, some of which are summarised in Table 1. This article focuses on pharmacological manipulations in relation to the influence of GnRH analogues.

\section{GnRH analogues and assisted reproductive techniques (ART) Controlled ovarian stimulation without $\mathrm{LH}$ surge suppression}

Before GnRHla was available, ovarian stimulation for IVF employed a standard regimen of the anti-oestrogen clomiphene citrate $(\mathrm{CC})$ and human menopausal gonadotrophin (hMG). The latter is a urinary derivative comprising of equivalent FSH/LH components (75 IU). Early extractions were compromised by a high degree (more than 90\%) of gonadotrophin-unrelated protein impurity. Multiple follicle recruitment was consistently achieved with high luteal phase progesterone levels obviating the need for progesterone supplementation. However, the risk of premature LH rise was ever present and led to the cancellation of $20-25 \%$ of cycles due to the positive feedback triggered by high serum oestrogen levels in the midfollicular phase. This led to oocyte maturation arrest or severely compromised IVF outcomes due to 'lost'/ovulated oocytes (11).

Apart from the generally well-tolerated systemic antioestrogenic side effects of CC (hot flushes occurring in 10\%, nausea, vomiting, mild skin reactions, breast tenderness, dizziness in less than $2 \%$ ), it also has an undesirable antioestrogenic effect on the endometrium. This is implicated in the discrepancy between relatively low embryo implantation rates following successful ovarian stimulation (12). Pregnancy rates were in the region of $6 \%$ per cycle started, and $20 \%$ per embryo transfer (13). For comparison, modern day practices would expect to achieve pregnancy rates of $20 \%$ and $30 \%$ respectively.

\section{Impact of different gonadotrophin preparations}

\section{Purity}

The degree of impurities in early hMG preparations required them to be administered by deep i.m. injection but they still gave rise to frequent unpleasant skin 
Table 1 Factors influencing the potential for IVF success

\begin{tabular}{|c|c|}
\hline Factor & Consequences \\
\hline Female age $(26,69)$ & $\begin{array}{l}\text { Accelerated decline in ovarian reserve after aged } 37 \text { years causes } \\
\text { reduced oocyte numbers and quality (increased miscarriage) }\end{array}$ \\
\hline \multicolumn{2}{|l|}{ Diagnosis of infertility } \\
\hline Tubal disease & $\begin{array}{l}\text { Tubal disease has a good prognosis primarily because oocyte quality and } \\
\text { reserve should be normal }\end{array}$ \\
\hline Male factor & $\begin{array}{l}\text { The treatment of male factor infertility was revolutionised by intracyto- } \\
\text { plasmic sperm injection. Once embryos have been created, pregnancy } \\
\text { rates are above average }\end{array}$ \\
\hline Ovulatory dysfunction & $\begin{array}{l}\text { Polycystic ovary syndrome is the commonest cause and is associated with } \\
\text { ovarian hyperstimulation (excessive recruitment of oocytes with many } \\
\text { being immature and without the potential to fertilise) }\end{array}$ \\
\hline Unexplained & $\begin{array}{l}\text { A diagnosis of exclusion encompassing many hypothetical problems or } \\
\text { combinations of problems }\end{array}$ \\
\hline Abnormal body weight/body mass index (70) & $\begin{array}{l}\text { Affects the endocrine milieu and pharmacodynamics and is a hazard in } \\
\text { pregnancy }\end{array}$ \\
\hline \multicolumn{2}{|l|}{ Pharmacological manipulations } \\
\hline $\begin{array}{l}\text { Preparation type, dose and duration of gonadotrophin } \\
\text { stimulation }\end{array}$ & $\begin{array}{l}\text { All may affect follicle dynamics, endometrial development, embryo quality, } \\
\text { risk of ovarian hyperstimulation syndrome (see text) }\end{array}$ \\
\hline Type of LH surge suppression (GnRH1a versus antagonist) & \\
\hline Type of ovulatory trigger & \\
\hline \multirow{2}{*}{\multicolumn{2}{|c|}{$\begin{array}{l}\text { Type, dose and duration of luteal support } \\
\text { Laboratory practices }\end{array}$}} \\
\hline & \\
\hline Culture medium, gamete and embryo handling and storage & $\begin{array}{l}\text { The conditions aim to replicate the physiological environment with minimal } \\
\text { handling but are inevitably artificial }\end{array}$ \\
\hline \multicolumn{2}{|l|}{ Technological aspects } \\
\hline Accurate ultrasound scan monitoring & $\begin{array}{l}\text { Many clinics now have nurse practitioners with these skills which can be } \\
\text { acquired easily with good training and supervision without compromis- } \\
\text { ing pregnancy rates or increasing complications }\end{array}$ \\
\hline $\begin{array}{l}\text { Accurate timing \& technique of oocyte retrieval } \\
\text { Accurate and atraumatic embryo transfer }\end{array}$ & \\
\hline
\end{tabular}

reactions and occasionally unpredictable ovarian response due to considerable batch to batch variability. By the 1980s, further improvements in extraction using monoclonal antibodies produced purified urinary FSH (uFSH) that generated much less hypersensitivity enabling s.c. administration. The relative LH content was negligible (5).

As the demand and provision of IVF soared, the pharmaceutical industry explored new avenues to meet the demand for gonadotrophins. Recombinant human FSH (rFSH) was the most complex protein ever produced from scratch. It requires a eukaryotic cell line with the intracellular mechanisms to achieve glycosylation. Consequently, there is now a limitless supply of consistently high purity, high biopotency product (follitropin $\alpha /$ Gonal F (Merck-Serono) and follitropin $\beta /$ Puregon (Organon)) without contamination with human-derived proteins of undetermined origins. These preparations have no LH activity at all.

\section{LH component}

The role of LH in follicle development has been the subject of much research and debate. According to the two-cell two-gonadotrophin concept, both FSH and LH are required for normal follicular development and steroidogenesis (14). LH binds to membrane-bound receptors on the theca cells of the ovary. This triggers an increase in steroidogenesis primarily of thecal androgen production. The androstenedione generated is subsequently aromatised to oestrogen in the granulosa cells (GCs) of the developing follicle. GCs that have been exposed to FSH also express LH receptors in the late follicular phase. In hypogonadotrophic women, ovulation induction without LH produces lower oestradiol concentrations and indeed no term pregnancies have been recorded with FSH alone in such patients. However, in normogonadotrophic women, several studies have shown that very low amounts of LH are sufficient for normal follicle and oocyte development (15). In addition, elevated follicular phase LH has been associated with reduced fertility and increased risk of miscarriage (16). This concept therefore has implications for the type of gonadotrophin preparation employed, and also the type and degree of pituitary suppression of endogenous LH production. Several studies have shown that in most women, endogenous levels of LH are sufficient for normal folliculogenesis despite pituitary downregulation and stimulation with pure FSH $(17,18)$.

There has been extensive research and meta-analysis into the potential differences between the three types of gonadotrophin preparations, much of it sponsored by the industry. Comparisons are hampered by variations in protocols (such as whether or not GnRH1a downregulation has been used) and outcome measures 
(e.g. oocyte numbers retrieved, clinical pregnancy rates, live birth rates). In the absence of GnRH1a downregulation, uFSH was more effective than hMG in terms of clinical pregnancy rates, but with downregulation, no differences in pregnancy rates were found (19). When rFSH was compared with hMG (after long protocol downregulation) in the recent meta-analysis of seven studies, hMG was associated with a significant $4 \%$ increase in live birth rate (95\% CI: 1-7\%) (20). The pooled number of participants exceeded 2000 and the findings withstood robust heterogeneity testing. A note of caution is sounded that although the findings were statistically significant, the confidence interval broached 1. Clinical significance is a matter of judgement for couples undergoing treatment and their clinicians.

Several studies have looked at LH supplementation in the late follicular phase in certain groups of patients. This could be beneficial by accelerating leading follicle development thereby decreasing the overall duration of treatment, particularly in those patients with a tendency to over-respond (hyperstimulate) with standard FSH stimulation (21). The concept of a 'window' for LH has arisen from findings that not only can LH stimulate GC proliferation and steroidogenesis but that in high doses it can cause follicle atresia or luteinisation. Much more research is needed to tease out the effect of LH supplementation in that group of normogonadotrophic women who might benefit from it.

\section{Dosage}

Starting doses of gonadotrophin for a first cycle of controlled stimulation vary between 100 and $300 \mathrm{IU} /$ day, depending on the patient's age and crude assessment of ovarian reserve (baseline follicular phase $\mathrm{FSH},+/-$ anti-mullerian hormone, + / - antral follicle count). Dose increases mid-cycle do not make a significant difference as the recruitment window of growing, receptive follicles has passed (22). The closest correlator determining the number of follicles recruited is the patient's age, which is related to the number of follicles in the current recruitable growth wave. This declines progressively and more rapidly after the age of 37 years (23). Reducing the dose of gonadotrophins, or withholding further stimulation (so-called coasting) in patients who have overstimulated, is commonly practiced but without conclusive evidence of efficacy (24).

\section{GnRH1 agonist and GnRH antagonist stimulation protocols}

\section{Agonists}

Although the advent of GnRH1a has had a significant impact on IVF outcome for two decades, there have been few dose finding studies or randomised controlled trials comparing the different agonists available (monthly depot injection, once daily s.c. injection and three times daily nasal administration). In 2005, The Cochrane Collaboration reported no significant differences in outcomes after depot versus daily GnRH1a administration (25). What has received more attention has been the optimal protocol in which to apply them. In the long protocol (Fig. 2a), GnRH1a is started in the luteal phase of the run-in cycle and continued until the ovulatory hCG trigger. Its agonistic mechanism means that there is an initial flare of gonadotrophin release before the receptors are downregulated. Stimulation usually commences about 2 weeks later, when the endometrium from the run-in cycle has been shed as well. There is flexibility in the scheduling at this stage as a few more days of downregulation do not affect the subsequent stimulation or cycle outcome to any significant degree (26). Some women will experience hypoestrogenic side effects of varying severity during this time. It is not clear whether the ovarian response to exogenous FSH is affected by GnRH1a although it is suspected to do so in some circumstances of reduced ovarian reserve.

The short or flare protocol (Fig. 2b) employs the agonist-induced flare up of endogenous FSH to stimulate the ovary in addition to exogenous FSH administration. The agonist is started on day 2 of the cycle with gonadotrophins on day 3. Follicular growth takes 10-12 days which is adequate to downregulate the pituitary and prevent a premature LH surge (27). There is little flexibility with this approach as the start of stimulation is dependent on the start of menses. Metaanalysis of short and long protocols has shown the long protocol to yield higher pregnancy rates from higher numbers of oocytes although more units of gonadotrophins are required (28). Commencement of GnRH1a in the mid-luteal phase of the preceding cycle is more successful than the follicular phase (when persistent follicular cysts tend to form) or any other time in the luteal half of the cycle $(29,30)$. One disadvantage is that an occult pregnancy may be present but no detrimental effects have been identified (31): menses fails to start and this should prompt testing for pregnancy.

\section{Antagonists}

The long protocol, supported by a wealth of evidence, has been widely adopted as the standard of care. However, it now faces a stern challenge from the newer antagonist protocols. Several studies have attempted to determine the minimum effective dose to achieve pituitary LH suppression and two strategies have emerged. A single depot may be used on cycle stimulation day 8 or 9 which lasts 4 days and is sufficient to prevent the LH surge in $80 \%$ of women (32) (Fig. 3a). Alternatively, multiple small doses may be used daily from cycle day 6 as a fixed order, or when the leading follicle has reached $14 \mathrm{~mm}$ diameter in a more flexible manner, until the hCG trigger $(33,34)$ (Fig. 3b). Initially, large prospective trials comparing the long agonist standard protocol with daily antagonist revealed 
(a) GnRH1 ant. bolus on D8

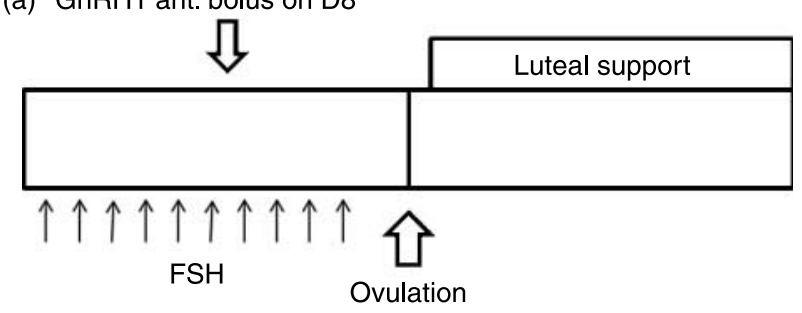

(b) GnRH1 ant. daily from D6

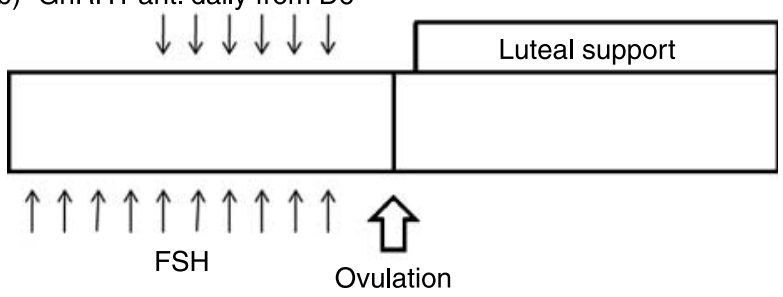

Figure $3 \mathrm{GnRH}$ antagonist protocols. a) GnRH antagonist cycle with $3 \mathrm{mg}$ bolus on days 8-9. b) GnRH antagonist cycle with $0.25 \mathrm{mg}$ daily from day 6 or when the lead follicle attains $14 \mathrm{~mm}$ diameter.

the latter to generate slightly fewer follicles, fewer oocytes and a decrement in pregnancy rate of 5\% (OR 0.75; 95\% CI 0.62-0.97) (35). Oocyte and embryo biology were equivalent with a good percentage of metaphase II oocytes, good fertilisation rates and good quality embryos. The better pregnancy rate in the agonist cycles was felt to be due to larger oocyte and embryo numbers affording greater selection for transfer. It has been suggested that the antagonist may have been started too early (day 6) in some patients with the consequence being reduced number and quality of embryos. If the start of the antagonist is delayed, the theory is that the window of FSH responsiveness is extended through the follicular phase, maximising potential recruitment.

In the initial dose-finding studies, there was an inverse relationship between antagonist dose and implantation rates suggesting a potentially embryotoxic effect (36). These concerns have not been borne out in frozen-thawed embryo cycles with the embryos resulting from antagonist cycles (37), and neither were there any differences in the pregnancy rates compared with frozen-thawed embryos from agonist cycles (38).

More recent data have been systematically reviewed to determine whether the probability of live birth is dependent on the type of analogue used and/or the type of protocol followed. A total of 22 randomised controlled trials involving 3176 subjects using both agonists and antagonists in various protocols were examined to find that no significant differences in live births between the two types of analogues could be identified and furthermore that this remained the case in the subgroup analyses for type of agonist/antagonist and for each protocol (39).

What is clear about the antagonist protocols is that they find greater favour with the patients. The treatment cycle length is more compact and physiological (essentially confined to a single month). The duration and dose of FSH is reduced. The antagonists are free of side effects and the risk of hospital admission due to hyperstimulation is reduced (40). It seems likely that antagonists will be increasingly adopted and demanded in a consumer-driven market. Service providers need to prepare to offer a seven-day service, which for some will be a somewhat retrograde step to the days before the agonists enabled some flexibility to workload scheduling.

One way around this problem, which was used before agonists superseded, is to use the combined oral contraceptive pill (COCP) to manipulate the length of the follicular phase. Some studies have found that prolonged pre-treatment is associated with excessive suppression, prolonged stimulation and increased cycle cancellation $(41,42)$. Others have not found any detrimental effects (43). Some encouraging work has been done on the pre-treatment of antagonist cycles (44) but much more is required.

\section{GnRH analogues and the second half of the cycle}

\section{Ovulation}

In natural cycles the LH surge begins the process of ovulation. Meiosis resumes leading to oocyte maturation in the preovulatory follicle. The follicle ruptures releasing the expanded cumulus-oocyte complex, leaving the residual follicle wall to convert into the corpus luteum (CL). Subsequently, pulsatile LH release promotes the continued development and normal lifespan of the CL. The exponential rise of hCG secreted by an implanting blastocyst and its trophoblast extends the CL lifespan until the placenta takes over the endocrine function. The purpose of GnRH analogues in IVF is to prevent the first part of this process but the subsequent functions of LH are still very much required. Agonists have a delayed onset of action and a delayed recovery and therefore the potential deficiency in luteal function must be rectified (see below). Conveniently, hCG, which is widely available and cheap has been used as an effective LH surrogate for many years. This is possible due to the shared $\alpha$-subunit.

Recombinant LH is also now available but has not achieved wide application as an ovulation trigger. It has been marketed more for use in endogenous LH deficiency such as hypogonadotrophic hypogonadism. Recombinant hCG is also available with its main advantage being the absence of potential prion contamination.

Agonists have also been applied successfully in antagonist cycles to bring about an endogenous LH surge capable of ovulation, demonstrating that the pituitary remains responsive under daily low dose antagonist influence (45). This strategy may prove to be beneficial for patients vulnerable to the protracted 
stimulatory effects of standard hCG on the over-stimulated ovary such as those with PCOS, who are at higher risk of ovarian hyperstimulation syndrome (OHSS)(46).

\section{Luteal phase}

CL function Luteal phase disruption has been recognised from the early days of IVF treatment. Progesterone levels are rapidly and markedly elevated in the early luteal phase of stimulated cycles but fall rapidly leading to significant shortening of this part of the cycle (47). The half life of the hCG used to trigger ovulation is long (much longer than the physiological LH surge), but not sufficient to sustain the CL for the duration required (48). Supplementary hCG has been used on days 3 and 5 after embryo transfer to 'rescue' CL progesterone production (49) and improved pregnancy rates result (50). One of the untoward side effects of hCG-triggered GC luteinisation is the enhanced production of vasoactive endothelial growth factor (VEGF). VEGF is important in the development and maintenance of vasculature in the developing tissue beds of the CL and decidua. However, it has also been implicated in the pathophysiology of OHSS, when it is overexpressed and increasingly bioavailable (51). The consequences can be severe with intravascular volume loss and extravascular fluid accumulation leading to thromboembolic disease, ascites, renal and cardiorespiratory compromise. Up to $5 \%$ of hCG-supported cycles are affected by significant OHSS (50). The risk is greater the more follicles have been stimulated, and particularly so in patients with polycystic ovary syndrome. Consequently, there has been a move away from using hCG after ovulation and most protocols use exogenous progesterone supplementation of the luteal phase instead (52).

Attempts to enable pituitary recovery by early cessation of GnRH1a have repeatedly failed $(53,54)$. However, it was not unreasonable to expect better pituitary recovery after antagonist suppression of ovulation because of the much shorter half-life. Unfortunately it is now clear that luteolysis is also initiated prematurely and pregnancy rates are compromised without the use of luteal support $(55,56)$.

The incidence of OHSS appears to be lower in metaanalysis following antagonist suppression compared with agonist cycles (OR 0.47; 95\% CI 0.18-1.25) (35).

\section{Endometrial receptivity}

The supraphysiological hormonal environment during IVF treatment is widely believed to be detrimental to the receptivity of the endometrium to implantation and supporting early pregnancy development. Elevated oestrogen concentrations generated during stimulation may increase the sensitivity of the endometrium to progesterone and promote secretory advancement. This has been demonstrated in endometrial biopsies taken before hCG injection, and therefore before exposure to progesterone (57). When the endometrium has been sampled on the day of oocyte retrieval, it has shown advancement in a high proportion of both agonist (58) and antagonist cycles (59). If advancement exceeds 3 days, pregnancy is unlikely (60).

\section{Embryo quality}

There is no evidence suggesting embryo quality is altered in relation to $\mathrm{GnRH}$ agonist or antagonist use but exogenous gonadotrophins have been shown to delay mammalian (mouse) embryo development between the 1-2 cell stage and blastocyst (61). There is some concerning evidence that exogenous gonadotrophin treatment increases the frequency of chromosomal abnormalities, demonstrated in preimplantation human embryos (62). Other work has failed to demonstrate a negative impact on embryo quality as a result of excessive ovarian stimulation (63), and another failed to identify any cleavage differences in embryos resulting from natural versus stimulated cycles (64).

\section{Contemporary issues in ART: increasing safer success, reducing multiples and OHSS}

\section{Poor responders}

Alterations to stimulation protocols are really the only strategy to try to improve outcomes for patients with a history of a poor response. Predicting a poor response is possible in the face of advanced age, elevated baseline FSH levels, low anti-mullerian hormone levels (a GC product of growing follicles) and/or low antral follicle counts on baseline ultrasound assessment of the ovaries. However, in the presence of normal parameters, and age under 40 years, a poor response to ovarian stimulation may be the first sign of ovarian aging, preceding any other abnormalities.

Increasing the dose of gonadotrophin stimulation is widely used within the range 100-450 IU daily, but there is little evidence to support higher doses $(22,65)$. Changing from a long agonist protocol to a flare has been demonstrated to reduce the incidence of cycle cancellation for poor response (66). The use of antagonists has been proposed to benefit this group of patients on the premise that they afford reduced suppression of endogenous gonadotrophins. Large trials are required to test this hypothesis, and particularly in relation to live birth outcomes. Improved oocyte numbers that do not translate into live births are unhelpful and give rise to false hope. 


\section{Mild stimulation}

After almost three decades of profound ovarian stimulation in the belief that a large number of oocytes is needed to compensate for subsequent inefficiencies in IVF treatment, there is now a move towards milder stimulation, driven by many of the factors addressed thus far: complex, expensive, long treatment cycles requiring intensive monitoring; medications with unpleasant and potentially serious side effects; abnormal endocrinology that is detrimental to the creation and establishment of normal pregnancy, including potential chromosomal aberration in the embryo.

As laboratory technology has improved and the ability to successfully freeze and thaw good quality embryos has evolved, in parallel with worldwide concerns regarding the consequences of multiple pregnancies (of which $40 \%$ in the UK are now the products of ART), these is a strong move towards reducing the number of embryos transferred in each cycle to a single one (67).

Following on from single embryo transfer is the vogue towards milder stimulation aiming for fewer oocytes and fewer embryos but of potentially better quality with potentially better prognosis of successful pregnancy (56, 68). A normal low response to low stimulation has greater prospects of success than an abnormal low response to maximal stimulation.

These strategies may reasonably be expected to substantially reduce multiple pregnancies and their significant health and economic impact, and also reduce the incidence of OHSS, the other serious complication of IVF treatment.

\section{Disclosure}

This paper forms part of a European Journal of Endocrinology supplement, supported by Ferring. The authors disclose no potential conflicting relationship with Ferring. This article was subject to rigorous peer review before acceptance and publication.

\section{References}

1 Ascheim S \& Zondek B. Hypophysenvorderlappen hormone und ovarialhormone im Harn von Schargeren. Klinische Wochenschrift 19276 13-21.

2 Fevold SL, Hisaw FL \& Leonard SL. The gonad-stimulating and the luteinizing hormones of the anterior lobe of the hypophysis. American Journal of Physiology 193197 291-301.

3 Hamblen EC. Endocrine therapy of functional ovarian failure. American Journal of Obstetrics and Gynecology 194040 615-662.

4 Gemzell CA. Induction of ovulation with human pituitary gonadotrophins. Fertility and Sterility 196213 153-168.

5 Hayden CJ, Balen AH \& Rutherford AJ. Recombinant gonadotrophins. British Journal of Obstetrics and Gynaecology 1999106 188-196.

6 Schally AV. Luteinizing hormone-releasing hormone analogs: their impact on the control of tumorigenesis. Peptides $1999 \mathbf{2 0}$ 1247-1262.

7 Conn PM \& Crowley WF Jr. Gonadotropin-releasing hormone and its analogs. Annual Review of Medicine $1994 \mathbf{4 5} 391-405$.
8 Leyendecker G, Wildt L \& Hansmann M. Pregnancies following chronic intermittent (pulsatile) administration of $\mathrm{Gn}-\mathrm{RH}$ by means of a portable pump ('Zyklomat') - a new approach to the treatment of infertility in hypothalamic amenorrhea. Journal of Clinical Endocrinology and Metabolism 198051 1214-1216.

9 Porter RN, Smith W, Craft IL, Abdulwahid NA \& Jacobs HS. Induction of ovulation for in vitro fertilisation using buserelin and gonadotropins. Lancet 19842 1284-1285.

10 Smitz J, Devroey P, Camus M, Deschacht J, Khan I, Staessen C, Van Waesberghe L, Wisanto A \& Van Steirteghem AC. The luteal phase and early pregnancy after combined GnRH-agonist/HMG treatment for superovulation in IVF or GIFT. Human Reproduction 1988 3 585-590.

11 Stanger JD \& Yovich JL. Reduced in vitro fertilization of human oocytes from patients with raised basal luteinizing hormone levels during the follicular phase. British Journal of Obstetrics and Gynaecology 198592 385-393.

12 Fauser BC \& Macklon NS. Medical approaches to ovarian stimulation for infertility. In Yen and Yaffe's Reproductive Endocrinology, pp 965-1012. Eds JF Strauss \& RL Barbieri, Philadelphia: Elsevier Saunders, 2004.

13 Kenny DT. In vitro fertilisation and gamete intrafallopian transfer: an integrative analysis of research, 1987-1992. British Journal of Obstetrics and Gynaecology 1995102 317-325.

14 Kobayashi M, Nakano R \& Ooshima A. Immunohistochemical localization of pituitary gonadotrophins and gonadal steroids confirms the 'two-cell, two-gonadotrophin' hypothesis of steroidogenesis in the human ovary. Journal of Endocrinology 1990126 483-488.

15 Chappel SC \& Howles C. Reevaluation of the roles of luteinizing hormone and follicle-stimulating hormone in the ovulatory process. Human Reproduction 19916 1206-1212.

16 Regan L, Owen EJ \& Jacobs HS. Hypersecretion of luteinising hormone, infertility, and miscarriage. Lancet $19903361141-1144$.

17 Frydman R, Howles CM \& Truong F. A double-blind, randomized study to compare recombinant human follicle stimulating hormone (FSH; Gonal-F) with highly purified urinary FSH (Metrodin) HP) in women undergoing assisted reproductive techniques including intracytoplasmic sperm injection. The French Multicentre Trialists. Human Reproduction 200015 520-525.

18 Daya $S$ \& Gunby J. Recombinant versus urinary follicle stimulating hormone for ovarian stimulation in assisted reproduction cycles. Cochrane Database of Systematic Reviews, 2000 (CD002810).

19 Agrawal R, Holmes J \& Jacobs HS. Follicle-stimulating hormone or human menopausal gonadotropin for ovarian stimulation in in vitro fertilization cycles: a meta-analysis. Fertility and Sterility 200073 338-343.

20 Coomarasamy A, Afnan M, Cheema D, van der Veen F, Bossuyt PM \& van Wely M. Urinary hMG versus recombinant FSH for controlled ovarian hyperstimulation following an agonist long downregulation protocol in IVF or ICSI treatment: a systematic review and meta-analysis. Human Reproduction 200823 310-315.

21 Hugues JN, Soussis J, Calderon I, Balasch J, Anderson RA \& Romeu A. Does the addition of recombinant LH in WHO group II anovulatory women over-responding to FSH treatment reduce the number of developing follicles? A dose-finding study Human Reproduction $2005 \mathbf{2 0} 629-635$.

22 van Hooff MH, Alberda AT, Huisman GJ, Zeilmaker GH \& Leerentveld RA. Doubling the human menopausal gonadotrophin dose in the course of an in vitro fertilization treatment cycle in low responders: a randomized study. Human Reproduction $1993 \mathbf{8}$ 369-373.

23 Faddy MJ, Gosden RG, Gougeon A, Richardson SJ \& Nelson JF. Accelerated disappearance of ovarian follicles in mid-life: implications for forecasting menopause. Human Reproduction 19927 1342-1346.

24 D'Angelo A \& Amso N. 'Coasting' (withholding gonadotrophins) for preventing ovarian hyperstimulation syndrome. Cochrane Database of Systematic Reviews, 2002 (CDO02811). 
25 Albuquerque LE, Saconato H \& Maciel MC. Depot versus daily administration of gonadotrophin releasing hormone agonist protocols for pituitary desensitization in assisted reproduction cycles. Cochrane Database of Systematic Reviews, 2005 (CD002808).

26 Chuang CC, Chen CD, Chao KH, Chen SU, Ho HN \& Yang YS. Age is a better predictor of pregnancy potential than basal folliclestimulating hormone levels in women undergoing in vitro fertilization. Fertility and Sterility 200379 63-68.

27 Smitz J, Devroey P \& Van Steirteghem AC. Endocrinology in luteal phase and implantation. British Medical Bulletin $1990 \mathbf{4 6}$ 709-719.

28 Daya S. Gonadotropin releasing hormone agonist protocols for pituitary desensitization in in vitro fertilization and gamete intrafallopian transfer cycles. Cochrane Database of Systematic Reviews, 2000 (CD001299).

29 Pellicer A, Simon C, Miro F, Castellvi RM, Ruiz A, Ruiz M, Perez M \& Bonilla-Musoles F. Ovarian response and outcome of in vitro fertilization in patients treated with gonadotrophin-releasing hormone analogues in different phases of the menstrual cycle. Human Reproduction $19894285-289$.

30 San Roman GA, Surrey ES, Judd HL \& Kerin JF. A prospective randomized comparison of luteal phase versus concurrent follicular phase initiation of gonadotropin-releasing hormone agonist for in vitro fertilization. Fertility and Sterility 199258 744-749.

31 Janssens RM, Brus L, Cahill DJ, Huirne JA, Schoemaker J \& Lambalk CB. Direct ovarian effects and safety aspects of GnRH agonists and antagonists. Human Reproduction Update 20006 505-518.

32 Olivennes F, Alvarez S, Bouchard P, Fanchin R, Salat-Baroux J \& Frydman R. The use of a GnRH antagonist (Cetrorelix) in a single dose protocol in IVF-embryo transfer: a dose finding study of 3 versus 2 mg. Human Reproduction 199813 2411-2414.

33 Albano C, Smitz J, Camus M, Riethmuller-Winzen H, Van Steirteghem A \& Devroey P. Comparison of different doses of gonadotropin-releasing hormone antagonist Cetrorelix during controlled ovarian hyperstimulation. Fertility and Sterility 1997 67 917-922.

34 The Ganirelix Dose-Finding Study Group. A double-blind, randomized, dose-finding study to assess the efficacy of the gonadotrophinreleasing hormone antagonist ganirelix (Org 37462) to prevent premature luteinizing hormone surges in women undergoing ovarian stimulation with recombinant follicle stimulating hormone (Puregon). Human Reproduction 199813 3023-3031.

35 Al-Inany H \& Aboulghar M. GnRH antagonist in assisted reproduction: a Cochrane review. Human Reproduction 200217 874-885.

36 Chian RC, Buckett WM, Tulandi T \& Tan SL. Prospective randomized study of human chorionic gonadotrophin priming before immature oocyte retrieval from unstimulated women with polycystic ovarian syndrome. Human Reproduction 200015 165-170.

37 Kol S, Lightman A, Hillensjo T, Devroey P, Fauser B, Tarlatzis B, Mannaerts B \& Itskovitz-Eldor J. High doses of gonadotrophinreleasing hormone antagonist in in vitro fertilization cycles do not adversely affect the outcome of subsequent freeze-thaw cycles. Human Reproduction $1999142242-2244$.

38 Seelig AS, Al-Hasani S, Katalinic A, Schopper B, Sturm R, Diedrich K \& Ludwig M. Comparison of cryopreservation outcome with gonadotropin-releasing hormone agonists or antagonists in the collecting cycle. Fertility and Sterility $200277472-475$.

39 Kolibianakis EM, Collins J, Tarlatzis BC, Devroey P, Diedrich K \& Griesinger G. Among patients treated for IVF with gonadotrophins and GnRH analogues, is the probability of live birth dependent on the type of analogue used? A systematic review and meta-analysis. Human Reproduction Update 200612 651-671.

40 Tarlatzis BC \& Kolibianakis EM. GnRH agonists vs antagonists. Best Practice \& Research. Clinical Obstetrics E Gynaecology 200721 57-65.

41 Mashiach S, Dor J, Goldenberg M, Shalev J, Blankstein J, Rudak E, Shoam Z, Finelt Z, Nebel L Goldman B et al. Protocols for induction of ovulation. The concept of programmed cycles. Annals of the New York Academy of Sciences $1988 \mathbf{5 4 1} 37-45$.
42 Benadiva CA, Ben-Rafael Z, Blasco L, Tureck R, Mastroianni L Jr \& Flickinger GL. An increased initial follicle-stimulating hormone/luteinizing hormone ratio does not affect ovarian responses and the outcomes of in vitro fertilization. Fertility and Sterility $1988 \mathbf{5 0}$ 777-781.

43 Gonen Y, Jacobson W \& Casper RF. Gonadotropin suppression with oral contraceptives before in vitro fertilization. Fertility and Sterility $199053282-287$.

44 van Loenen AC, Huirne JA, Schats R, Hompes PG \& Lambalk CB. GnRH agonists, antagonists, and assisted conception. Seminars in Reproductive Medicine 200220 349-364.

45 Felberbaum RE, Reissmann T, Kupker W, Bauer O, al Hasani S, Diedrich C \& Diedrich K. Preserved pituitary response under ovarian stimulation with HMG and GnRH antagonists (Cetrorelix) in women with tubal infertility. European Journal of Obstetrics, Gynecology, and Reproductive Biology $19956 \mathbf{6 1}$ 151-155.

46 Shalev E, Geslevich Y \& Ben-Ami M. Induction of pre-ovulatory luteinizing hormone surge by gonadotrophin-releasing hormone agonist for women at risk for developing the ovarian hyperstimulation syndrome. Human Reproduction 19949 417-419.

47 Jones HW Jr. What has happened? Where are we? Human Reproduction 199611 7-24 (discussion 29-31).

48 Mannaerts BM, Geurts TB \& Odink J. A randomized three-way cross-over study in healthy pituitary-suppressed women to compare the bioavailability of human chorionic gonadotrophin (Pregnyl) after intramuscular and subcutaneous administration. Human Reproduction 199813 1461-1464.

49 Herman A, Ron-El R, Golan A, Raziel A, Soffer Y \& Caspi E. Pregnancy rate and ovarian hyperstimulation after luteal human chorionic gonadotropin in in vitro fertilization stimulated with gonadotropin-releasing hormone analog and menotropins. Fertility and Sterility 199053 92-96.

50 Soliman S, Daya S, Collins J \& Hughes EG. The role of luteal phase support in infertility treatment: a meta-analysis of randomized trials. Fertility and Sterility 199461 1068-1076.

51 Levin ER, Rosen GF, Cassidenti DL, Yee B, Meldrum D, Wisot A \& Pedram A. Role of vascular endothelial cell growth factor in ovarian hyperstimulation syndrome. Journal of Clinical Investigation $1998 \mathbf{1 0 2} 1978-1985$.

52 Penzias AS. Luteal phase support. Fertility and Sterility 200277 318-323.

53 Beckers NG, Laven JS, Eijkemans MJ \& Fauser BC. Follicular and luteal phase characteristics following early cessation of gonadotrophin-releasing hormone agonist during ovarian stimulation for in vitro fertilization. Human Reproduction 200015 43-49.

54 Pantos K, Meimeth-Damianaki T, Vaxevanoglou T \& Kapetanakis E. Prospective study of a modified gonadotropinreleasing hormone agonist long protocol in an in vitro fertilization program. Fertility and Sterility 199461 709-713.

55 Albano C, Grimbizis G, Smitz J, Riethmuller-Winzen H, Reissmann T, Van Steirteghem A \& Devroey P. The luteal phase of nonsupplemented cycles after ovarian superovulation with human menopausal gonadotropin and the gonadotropin-releasing hormone antagonist Cetrorelix. Fertility and Sterility $1998 \mathbf{7 0}$ 357-359.

56 de Jong D, Macklon NS \& Fauser BC. A pilot study involving minimal ovarian stimulation for in vitro fertilization: extending the 'follicle-stimulating hormone window' combined with the gonadotropin-releasing hormone antagonist cetrorelix. Fertility and Sterility 200073 1051-1054.

57 Marchini M, Fedele L, Bianchi S, Losa GA, Ghisletta M \& Candiani GB. Secretory changes in preovulatory endometrium during controlled ovarian hyperstimulation with buserelin acetate and human gonadotropins. Fertility and Sterility $1991 \mathbf{5 5}$ 717-721.

58 Ubaldi F, Bourgain C, Tournaye H, Smitz J. Van Steirteghem A \& Devroey P. Endometrial evaluation by aspiration biopsy on the day of oocyte retrieval in the embryo transfer cycles in patients with serum progesterone rise during the follicular phase. Fertility and Sterility 199767 521-526. 
59 Kolibianakis E, Bourgain C, Albano C, Osmanagaoglu K, Smitz J, Van Steirteghem A \& Devroey P. Effect of ovarian stimulation with recombinant follicle-stimulating hormone, gonadotropin releasing hormone antagonists, and human chorionic gonadotropin on endometrial maturation on the day of oocyte pick-up. Fertility and Sterility 200278 1025-1029.

60 Devroey P, Bourgain C, Macklon NS \& Fauser BC. Reproductive biology and IVF: ovarian stimulation and endometrial receptivity. Trends in Endocrinology and Metabolism 200415 84-90.

61 Van der Auwera I \& D’Hooghe T. Superovulation of female mice delays embryonic and fetal development. Human Reproduction 200116 1237-1243.

62 Baart EB, Martini E, Eijkemans MJ, Van Opstal D, Beckers NG, Verhoeff A, Macklon NS \& Fauser BC. Milder ovarian stimulation for in vitro fertilization reduces aneuploidy in the human preimplantation embryo: a randomized controlled trial. Human Reproduction 200722 980-988.

63 Ng EH, Lau EY, Yeung WS \& Ho PC. Oocyte and embryo quality in patients with excessive ovarian response during in vitro fertilization treatment. Journal of Assisted Reproduction and Genetics 2003 20 186-191.

64 Ziebe S, Bangsboll S, Schmidt KL, Loft A, Lindhard A \& Nyboe Andersen A. Embryo quality in natural versus stimulated IVF cycles. Human Reproduction 200419 1457-1460.
65 Tarlatzis BC, Zepiridis L, Grimbizis G \& Bontis J. Clinical management of low ovarian response to stimulation for IVF: a systematic review. Human Reproduction Update 20039 61-76.

66 Surrey ES, Bower J, Hill DM, Ramsey J \& Surrey MW. Clinical and endocrine effects of a microdose $\mathrm{GnRH}$ agonist flare regimen administered to poor responders who are undergoing in vitro fertilization. Fertility and Sterility $199869419-424$.

67 Ledger WL, Anumba D, Marlow N, Thomas CM \& Wilson EC. The costs to the NHS of multiple births after IVF treatment in the UK. British Journal of Obstetrics and Gynaecology 2006113 21-25.

68 Macklon NS \& Fauser BC. Regulation of follicle development and novel approaches to ovarian stimulation for IVF. Human Reproduction Update 20006 307-312.

69 Gougeon A, Ecochard R \& Thalabard JC. Age-related changes of the population of human ovarian follicles: increase in the disappearance rate of non-growing and early-growing follicles in aging women. Biology of Reproduction 199450 653-663.

70 Group TECW. Nutrition and reproduction in women. Human Reproduction Update 200612 193-207.

Received 9 October 2008

Accepted 9 October 2008 